\title{
Psychophysiologic stress reduction in surgery
}

\author{
Annika Rieger $\cdot$ Regina Stoll
}

Received: 9 January 2012 / Accepted: 10 February 2012/Published online: 18 July 2012

(C) Springer Science+Business Media, LLC 2012

We read with interest the paper entitled Effects of Intraoperative Breaks on Mental and Somatic Operator Fatigue. This paper describes a randomized clinical trial authored by Engelmann and colleagues, who noted that "work breaks during complex laparoscopic surgery can reduce psychological stress and preserve performance without prolongation of the operation time compared with the traditional work scheme" [1].

Similar to the working group of Ure, our research team conducted a psychophysiologic study including 25 surgeons, who accomplished 51 surgeries. The volunteers were monitored over the period of 1 working day with a real-time working telemedical system [2]. The strain markers in our study included heart rate (HR), breathing rate (BR), skin temperature (ST), and three self-report questionnaires answered on a mobile phone before and after each surgery.

After the procedures, intraoperative function (primary surgeon, first assistant surgeon), level of complication, and application of lead aprons were documented on the mobile phone. Our results clearly indicated that the function during surgery and the wearing of lead aprons had an impact on the intraoperative stress of the surgeon. Surgeons in the function of primary operator showed higher levels of HR, $\mathrm{BR}$, and ST than they experienced in their role as first assistant surgeon. Furthermore, an additional increase in load was documented during operations with lead aprons.

The answers given in the self-report questionnaires supported the findings. The primary surgeons evaluated the intraoperative stress significantly higher than the first assistant surgeons, and the application of lead aprons also resulted in higher stress levels.

For this reason, we suggest that apart from consideration of intraoperative work breaks within one surgery, careful preoperative planning of consecutive surgeries that allows for both an alternation of intraoperative functions and a rotative system for the application of lead aprons should be taken into account.

\section{References}

1. Engelmann C, Schneider M, Kirschbaum C et al (2011) Effects of intraoperative breaks on mental and somatic operator fatigue: a randomized clinical trial. Surg Endosc Other Interv Tech 25: $1245-1250$

2. Neubert S, Arndt D, Thurow K et al (2010) Mobile real-time data acquisition system for application in preventive medicine. Telemed e-Health 16:504-509

\footnotetext{
A. Rieger $(\bowtie) \cdot R$. Stoll

Rostock University Medical Center, Institute for Preventive

Medicine, St.-Georg-Strasse 108, 18055 Rostock, Germany

e-mail: annika.rieger@uni-rostock.de

R. Stoll

e-mail: regina.stoll@uni-rostock.de
} 